\title{
ENTEROPARASITAS E PROFILAXIA EM ALUNOS DA ZONA RURAL DE SERRA TALHADA- PERNAMBUCO
}

\author{
Renan do Nascimento Barbosa \\ Bacharel em Ciências Biológicas \\ Universidade Federal Rural de Pernambuco (UFRPE) \\ e-mail: renan.rnb@gmail.com
}

\section{Bárbara Augusta Estima Mota}

Técnica em Meio Ambiente

Instituto Federal da Paraíba (IFPB)

e-mail: bmotacavalcanti@gmail.com

\section{Miriam Dowell de Brito Cavalcanti}

Especialista em Zoologia

Universidade Federal Rural de Pernambuco (UFRPE)

e-mail: dowell_miriam@yahoo.com.br

\section{Magnólia da Conceição Nunes Botêlho}

Especialista em Ectoparasitos e em Mídias na Educação (UFRPE)

Governo do Estado de Pernambuco

e-mail: magbotelho@yahoo.com.br

\section{Ednilza Maranhão dos Santos}

Doutora em Psicobiologia

Universidade Federal Rural de Pernambuco (UFRPE)

e-mail: ednilzamaranhao.gmail.com

Recebido: 20 de abril de 2013. Revisado: 27 de maio de 2013. Aceito: 15 de junho de 2013. Publicado online: 17 de julho de 2013

\section{RESUMO}

Este estudo teve como objetivo verificar a ocorrência de enteroparasitas em amostras fecais provenientes de crianças que estudam em uma escola na zona rural da cidade de Serra Talhada - Pernambuco, bem como realizar atividades de profilaxia. Três amostras fecais de 29 crianças foram coletadas e processada pelos métodos direto e de Hoffmann, Pons e Janer. Entamoeba coli foi o protozoário mais prevalente $(30,1 \%)$ seguido da Entamoeba histolytical dispar (28,5\%). Os helmintos detectados foram: Hymenolepis nana $(57,1 \%)$, Ancilostomideo $(42,9 \%)$. As atividades de profilaxia foram interativas envolvendo o lúdico, sendo consideradas satisfatórias, sugerindo-se um programa de educação continuada.

Palavras-chave: Crianças; Educação; Parasitoses.

\begin{abstract}
This study aimed to verify the occurrence of intestinal parasites in stool samples from children studying in a school in countryside of Serra Talhada - Pernambuco, as well as perform activities of prophylaxis. Three
\end{abstract}


stool samples from 29 children were collected and analyzed by the methods of direct and Hoffmann, Pons and Janer. Entamoeba coli was the most common protozoan (30,1\%), followed by Entamoeba histolytica/ dispar (28,5\%). The helminths found were Hymenolepis nana (57,1\%), Ancilostomideo (42,9\%). The activities were interactive prophylaxis involving playful. The activities were interactive prophylaxis involving the playful, being considered satisfactory, and suggested a program of continuing education.

Keywords: Children, Education, Parasites.

\section{INTRODUÇÃO}

As enteroparasitoses representam um grave problema de saúde pública em diversos países, em especial, naqueles em desenvolvimento como o Brasil, ocasionado danos no desempenho físico e cognitivo dos indivíduos acometidos (CASTRO et al.,2004; BARÇANTE et al., 2007), principalmente na capacidade em aprender conceitos, na velocidade do processamento de informações, na concentração e habilidade perceptiva, bem como na resolução de problemas (BOTELHO, 2008). Vários estudos revelam esse déficit, principalmente quando oriundo de infecções helmínticas em crianças de idade escolar (EZEAMAMA et al., 2005; SATKI et al., 1999; HADIDJAJA et al., 1998; NOKES et al., 1992).

Estudos indicam que as infecções parasitárias estão relacionadas as condições ambientais em que o indivíduo vive, entre elas aquelas relacionadas ao clima e alimentação, bem como ao saneamento básico e hábitos de higiene precários (COURA et al., 1993; VINHA, 1965; ULUKANLIGIL et al., 2001). Ao estudarem a distribuição de Ascaris lumbricoides no Rio de Janeiro, Fortes et al. (2004), observaram maior risco de contaminação entre os indivíduos que residiam em domicílios com mais de um morador por cômodo, com coleta de lixo irregular, falta de água filtrada, baixa renda familiar e dona de casa apresentando baixo nível de escolaridade (FORTES et al., 2004).

As parasitoses têm uma maior ocorrência entre os indivíduos em idade escolar, principalmente crianças, pois estas apresentam inconscientemente, hábitos precários de higiene, como levar as mãos sujas à boca. Desta forma, o diagnóstico através do exame parasitológico de fezes, é indispensável para melhor precisão na avaliação dos enteroparasitas e na prescrição dos diferentes agentes terapêuticos utilizados. Assim, é necessário identificar, tratar e prevenir as infecções parasitárias, a fím de evitar prováveis epidemias e formação de novas áreas endêmicas (PITTNER et al., 2006).

As relações dessas enfermidades com a comunidade, sobretudo com o homem do campo e populações de baixa renda, vêm chamando a atenção da sociedade brasileira desde a década de trinta, quando o escritor Monteiro Lobato lançou o ícone do indivíduo parasitado através do personagem do Jeca Tatu, que tem sua história como ferramenta educativa para problemas sanitários, principalmente para crianças, sendo contada ou adaptada para outros meios de comunicação, colaborando com o processo de conscientização (NEVES et al., 2005). Com isso, diagnósticos sociais e ações educativas/preventivas são importantes para a sensibilização dessas comunidades, a fim de que se obtenham resultados satisfatórios nas ações corretivas de seus hábitos de higiene, que resultam em parasitoses comuns nesse tipo de contexto. No caso de crianças em idade escolar, torna-se mais fácil chegar à família através das ações educativas vivenciadas no ambiente escolar.

O presente estudo teve por objetivo avaliar a prevalência de enteroparasitas e realizar atividades de sensibilização e conscientização através da educação ambiental em crianças de uma comunidade escolar da zona rural do município de Serra Talhada - PE.

\section{METODOLOGIA}

Este trabalho foi realizado no período de 16 de maio de 2007 a 07 de agosto de 2008, com crianças que estudam na $3^{\mathrm{a}}$ e $4^{\mathrm{a}}$ série do ensino fundamental de uma escola da zona rural, na cidade de Serra Talhada - PE. Os critérios utilizados na seleção dos alunos foram a faixa etária e o rendimento escolar. 
Após orientação aos responsáveis pelas crianças, através de reuniões e palestras, bem como o consentimento dos mesmos por meio do Termo de Consentimento Livre e Esclarecido (TCLE), as amostras de fezes foram coletadas durante três dias consecutivos, em potes coletores de plástico, contendo $20 \mathrm{ml}$ de solução formalina a 5\% como conservante, seguindo a metodologia de Moura et al., (2006). Os potes coletores foram recolhidos e encaminhados ao Laboratório de Parasitologia do Departamento de Biologia da UFRPE para realização de exames coproparasitológicos, onde foram utilizadas duas técnicas: Método Direto, de acordo com a descrição de Huggins et al., (1993); Método de Hoffman, Pons e Janner ou Sedimentação Espontânea, seguindo a metodologia de Evangelista et al., (1972), Coelho (1975) e De Carli et al., (1989).

As atividades multidisciplinares de profilaxia foram desenvolvidas por meio de técnicas que envolviam o lúdico e a contextualização como brincadeiras, jogos e palestras com diferentes temas: "Os bichinhos que causam danos a saúde, seus ciclos de vida e os prejuízos que eles causam", "Lavar as mãos antes das refeições", "Utilizar lugares longes de nascentes e corpos d'água para eliminar fezes", "Importância de cortar as unhas", "Beber água filtrada e fervida" entre outros. Todas as atividades orientavam as crianças para a adequação de hábitos higiênicos, com base na realidade local.

Cartazes informativos também foram elaborados pela equipe e distribuídos em pontos estratégicos na escola como bebedouros, banheiros, cozinha, salas de aulas e pátio. Foram utilizados como materiais didáticos durante as atividades: projetor de slides, vídeo, cartazes, lápis de cor, papel e modelos de parasitas, confeccionados pela equipe com massa de modelar. Os encontros educativos ocorriam tanto nas dependências da escola como na Universidade Federal Rural de Pernambuco/ Unidade Acadêmica de Serra Talhada (UAST).

Além das atividades dinâmicas, houve também a aplicação de um questionário com seis perguntas, adequando a linguagem científica à popular, envolvendo os aspectos de etiologia, ciclo evolutivo, transmissão, sintomatologia, diagnóstico e profilaxia. Foi solicitado aos alunos que respondessem as questões, sempre que possível, através de desenhos, uma vez que, as imagens são elementos que também constituem uma linguagem e ganham cada vez mais ênfase e importância no âmbito escolar, além de proporcionar maior compreensão dos conceitos abordados nas mais diversas disciplinas (MAIA e SCHIMIN, [s.d.]). Os critérios de avaliação das respostas foram baseados nos conceitos de Neves (2005) e Rey (1991), procurando analisar os resultados de acordo com o conhecimento científico correto transmitido pelos alunos.

\section{RESULTADOS E DISCUSSÃO}

\subsection{Pesquisa coproparasitológica}

Dos 40 alunos que frequentavam a escola, foram identificados 29 com baixo rendimento escolar, provavelmente devido a alguma deficiência de atenção.

Foram analisadas 29 amostras de fezes. Dessas, $100 \%$ estavam contaminadas por enteroparasitas, sendo $24,14 \%$ monoparasitadas e $75,86 \%$ poliparasitadas. Com os resultados pôde-se observar que crianças com dez anos de idade foram as mais acometidas, com a presença tanto de protozoários quanto de helmintos (Tabela 1).

Sete espécies de enteroparasitos foram identificadas, sendo Entamoeba coli $(30,1 \%)$ seguido por Entamoeba histolitica $(28,5 \%)$ os protozoários mais prevalentes. Esses resultados corroboram o trabalho de Correia et al., (2005). Dentre os helmintos, as ocorrências foram de Hymenolepis nana (57,1\%) seguido por Ancilostomídeos (42,9\%) (Tabela 1). 
Tabela I: Frequência absoluta e relativa dos enteroparasitas diagnosticados entre alunos da $3^{\mathrm{a}}$ e $4^{\mathrm{a}}$ série distribuídos entre as idades de nove e 15 anos. Fonte: Os autores.

\begin{tabular}{|c|c|c|c|c|c|c|c|c|}
\hline \multirow{3}{*}{ Enteroparasitas } & \multicolumn{7}{|c|}{ Idade das crianças } & \multirow{3}{*}{$\begin{array}{l}\text { Frequência } \\
\text { relativa }\end{array}$} \\
\hline & $\begin{array}{c}9 \\
\text { anos }\end{array}$ & $\begin{array}{c}10 \\
\text { anos }\end{array}$ & $\begin{array}{c}11 \\
\text { anos }\end{array}$ & $\begin{array}{c}12 \\
\text { anos } \\
\end{array}$ & $\begin{array}{c}13 \\
\text { anos } \\
\end{array}$ & $\begin{array}{c}14 \\
\text { anos } \\
\end{array}$ & $\begin{array}{c}15 \\
\text { anos } \\
\end{array}$ & \\
\hline & \multicolumn{7}{|c|}{ Frequência absoluta } & \\
\hline \multicolumn{9}{|l|}{ Protozoários } \\
\hline Giardia lamblia & 1 & 2 & 1 & - & - & - & 1 & $7,9 \%$ \\
\hline Endolimax nana & 1 & 7 & 1 & 1 & 2 & - & - & $19 \%$ \\
\hline $\begin{array}{l}\text { Blastocystis } \\
\text { hominis }\end{array}$ & 1 & 5 & - & & 2 & 1 & - & $14,2 \%$ \\
\hline $\begin{array}{l}\text { Entamoeba } \\
\text { histolytica/dispar }\end{array}$ & 3 & 5 & 2 & 3 & 3 & 1 & 1 & $28,5 \%$ \\
\hline Entamoeba coli & 4 & 7 & 2 & 1 & 3 & 1 & 1 & $30,1 \%$ \\
\hline \multicolumn{9}{|l|}{ Helmintos } \\
\hline Ancilostomideo & 1 & - & - & 1 & 1 & - & - & $42,9 \%$ \\
\hline Hymenolepis nana & 1 & 2 & - & - & 1 & - & - & $57,1 \%$ \\
\hline
\end{tabular}

Os resultados individuais dos exames foram entregues ao (s) responsável (is) dos alunos e os mesmos foram encaminhados ao posto de saúde da comunidade para que o medicamento adequado fosse prescrito e fornecido.

Em um estudo feito por Silva et al., (2009), a prevalência de enteroparasitoses foi observada em crianças residentes em comunidades rurais do município de Coari, estado do Amazonas, Brasil. Foram realizados exames coproparasitológicos em 123 crianças, obtendo-se o índice de $74 \%$ de amostras positivas. Os agentes etiológicos mais frequentes foram: A. lumbricoides (68,1\%), Trichuris trichiura (41,8\%), ancilostomatídeos (17,6\%), E. histolytica/E. dispar (17,6\%), E. coli (11\%), Giardia duodenalis (9,9\%), Strongyloides stercoralis (8,8\%), E. nana (3,3\%) e Iodameba butschilii (1,1\%).

Pittner et al., (2006), realizaram um estudo com 203 crianças pertencentes à Comunidade Escolar Guaratu, no município de Guarapuava- PR. Observaram que $123(60,59 \%)$ crianças foram positivas para pelo menos um parasito e a ocorrência de casos de poliparasitismo foi de $23,58 \%$, dos quais foram registrados maiores índices de infecções por Giardia intestinalis 50,73\% e A. lumbricoides 15,27\%. Este helminto tem sido frequentemente reportado em estudos coproparasitológicos em diferentes comunidades no Brasil (SEIXAS et al., 2011).

Como também observado por Seixas et al., (2011), embora amebas comensais tenham sido observadas, como E. nana e E. coli, ressalta-se que a transmissão ocorre da mesma forma que os outros mecanismos de transmissão de outros protozoários patogênicos, como E. Histolytica e E. dispar, servindo, desta forma, como importantes indicadores das condições sanitárias que os indivíduos convivem.

Mediante aos resultados obtidos, constatou-se que as crianças avaliadas apresentaram altos índices de enteroparasitas, corroborando os estudos acima citados. A elevada prevalência de enteroparasitos na comunidade estudada demonstra a dificuldade de controlar estas infecções e indica a necessidade de medidas preventivas e educativas adequadas à realidade de cada região. 


\subsection{Atividades educativas - profilaxia}

As interações com os alunos foram avaliadas como satisfatórias. Todas as exposições referentes ao conteúdo foram dinâmicas, onde as crianças puderam manipular e observar as estruturas dos parasitos, vetores e ovos em três dimensões durante as práticas com os modelos confeccionados com massa de modelar. Com as atividades (de escrita, desenhos, brincadeiras e jogos) foi possível observar que o conteúdo foi bem assimilado. Em relação aos desenhos e com os modelos ilustrativos de massa de modelar, observouse que a identificação dos parasitos foi feita de maneira correta, salvo, quando utilizaram expressões consideradas rotineiras para o público, por exemplo, "minhoca que vive na barriga" para o A. lumbricoides.

Os jogos e brincadeiras realizadas no pátio da escola e da UFRPE/UAST foram as atividades com maior participação, principalmente daquelas crianças com idade entre 4 e 10 anos. $\mathrm{O}$ conteúdo dos jogos e brincadeiras, como a de "pagar prendas" caso a resposta estivesse incorreta, tinham a temática voltada para a preservação ambiental, higiene pessoal e profilaxia das parasitoses. As práticas eram esperadas por todos e desfrutadas com bastante empolgação. Os cartazes também foram peça importante para a implementação das medidas de higiene (Fig. 1).

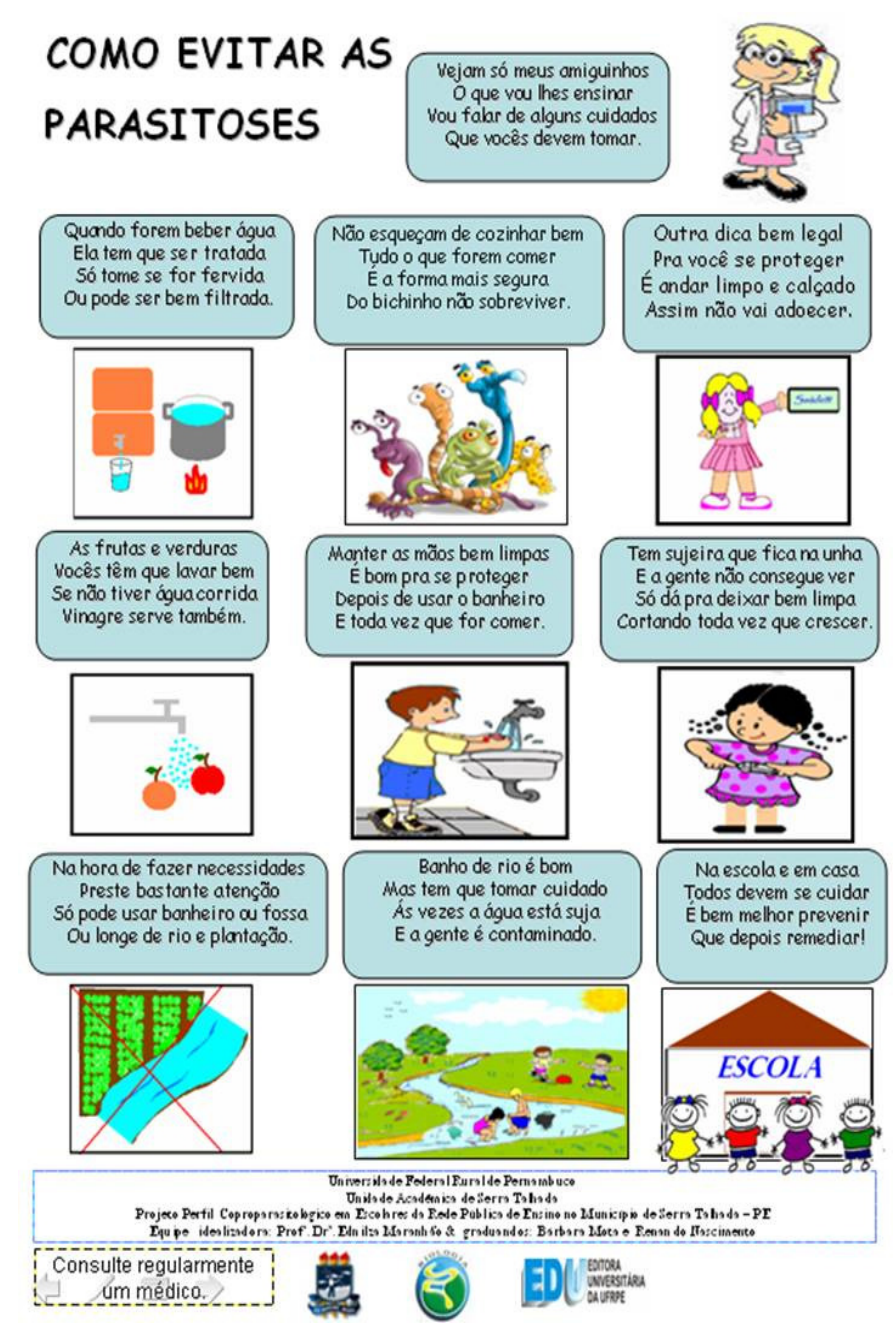

Figura 1: Cartaz informativo sobre as parasitoses, confeccionado pela equipe e disponibilizado a escola. Fonte das imagens: Google Imagem e banco de dados dos autores. Montagem e organização: Os autores.

Nas reuniões marcadas com os pais, apesar da pouca participação, observou-se que aqueles que estavam presentes reconheciam a importância da realização de projetos com esse foco na comunidade da zona rural. Os mesmos tiraram suas dúvidas acerca das parasitoses, principalmente em relação à profilaxia $\mathrm{e}$ 
melhor condição da água para consumo. Apesar do nível de instrução (a maioria não tinha terminado o ensino fundamental), foi perceptível o reconhecimento de alguns deles quando falavam das "doenças de hoje em dia" relacionando ao desequilíbrio ambiental.

Em relação aos questionários, pôde-se constatar que os alunos não possuiam um conceito bem definido sobre parasitoses, isso ficou evidente com os desenhos/respostas da primeira pergunta do questionário: “O que é uma verminose?". Foi abordada através do desenho que: são simplesmente vermes ou lombrigas (Fig. 2A). Siqueira e Fiorini (1999) encontraram resultados semelhantes em seus estudos.

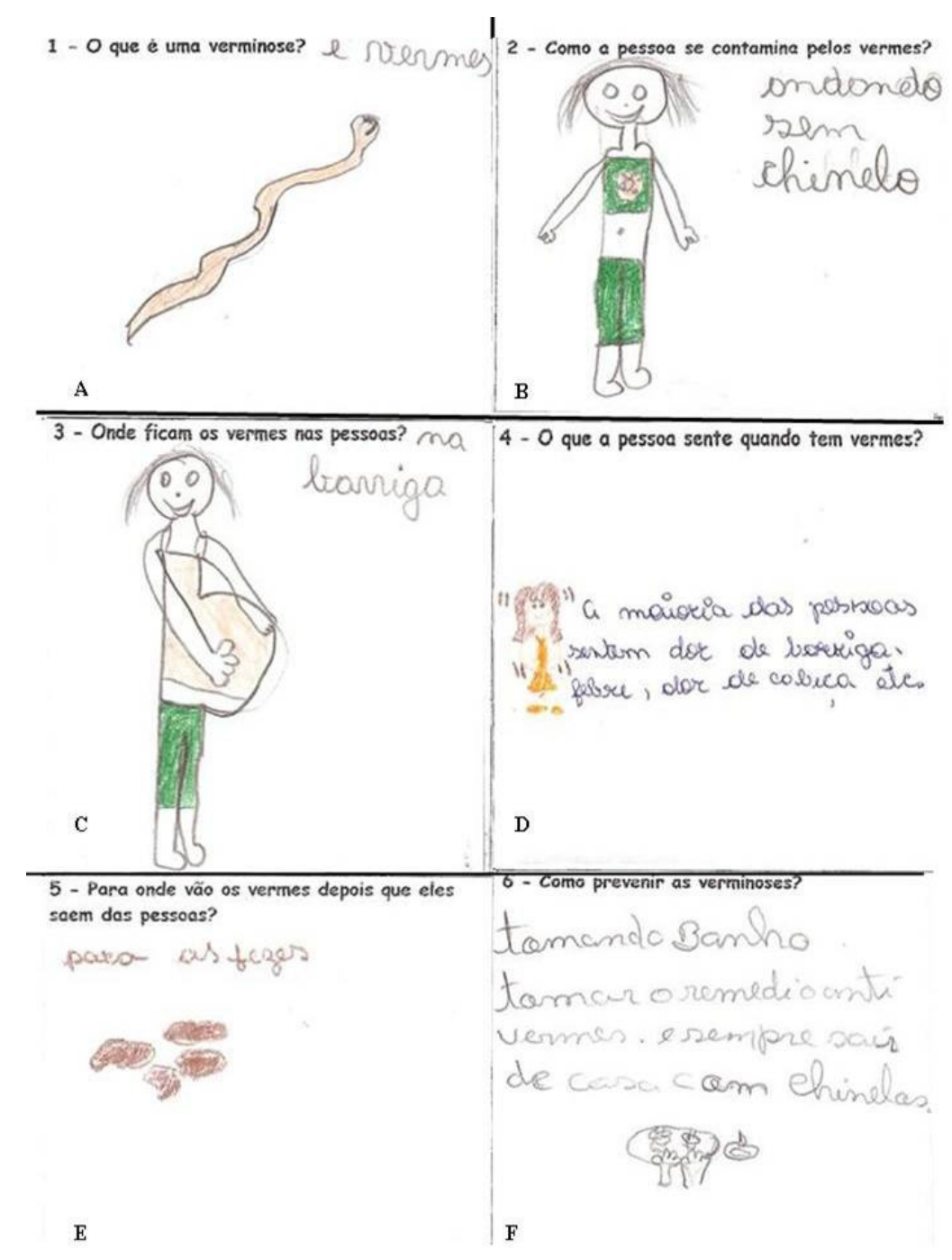

Figura 2: Montagem com alguns dos desenhos/resposta produzidos pelos alunos durante a pesquisa. AObserva-se a representação de um verme para a definição da primeira pergunta "O que é uma verminose?"; B- Ilustração de uma menina andando sem calçados como resposta dada para a segunda questão "Como a pessoa se contamina pelos vermes?"; C- Destaque na representação dada a barriga da pessoa representada pra indicar, além da resposta escrita, que os vermes ficam na barriga; D- Respostas dada para a pergunta “ $\mathrm{O}$ que a pessoa sente quando tem vermes?"; E- Ilustração de material fecal dada para a questão "Para onde vão os vermes depois que eles saem das pessoas?"; F- Destaque para a escrita, única resposta com indicativo de uso de vermicidas, além de medidas de higiene. Fonte: os autores.

Em relação a $2^{a}$ pergunta "Como a pessoa se contamina pelos vermes?" $50 \%$ dos alunos indicaram o hábito de andar descalço como a principal forma de contaminação das verminoses. Na $3^{\text {a }}$ questão "Onde ficam os vermes nas pessoas?", 76,2\% dos alunos indicaram que os vermes parasitam o organismo humano principalmente na região abdominal, utilizando ora o termo ora a ilustração de uma barriga grande (Fig.2C); apenas $9,52 \%$ dos alunos ilustraram/escreveram que os mesmos encontram-se principalmente no intestino, $\mathrm{o}$ 
que deixa claro que a localização das espécies parasitárias no organismo humano não é bem compreendida pela grande maioria dos entrevistados, não corroborando aos dados encontrados por Mello et al. (1988) e Siqueira e Fiorini (1999).

As respostas relacionadas à sintomatologia, abordadas na $4^{\text {a }}$ questão, indicam dores abdominais, febre, dor de cabeça e fraqueza. Foram os sintomas mais representados (Fig. 2D). Quando perguntado "Para onde vão os parasitas depois que eles saem das pessoas?" 73,8\% das ilustrações eram relacionadas a material fecal ou escreveram os termos "fezes" ou "cocô" (Fig. 2E). Apenas 7,1\% das respostas deram indicativos de contaminação ambiental. As medidas profiláticas com maior representação foram situações que retratavam o hábito de higiene como andar com calçados, não brincar com areia/terra, lavar as mãos e higiene com alimentos e água, evidenciando o conhecimento profilático revelado pelos alunos. A maioria sabe que medidas simples como, lavar as mãos antes das refeições e após defecar pode prevenir a contaminação, similar aos achados de Gomes dos Santos et al., (1993). Encontrou-se também, algumas respostas indicando erroneamente que uma das formas de evitar contaminação seria não comer coisas estragadas e doces. Diferentemente dos resultados encontrados por Uemura e Carvalho (1989), neste estudo houve apenas uma citação do uso de vermicidas (Fig. 2F).

Com base nos resultados deste trabalho, observou-se que os escolares apesar de terem alguns conhecimentos prévios sobre o tema abordado, mostraram dificuldades em relação à localização e a forma em que esses parasitas são transmitidos ao ser humano. Compreendemos, que é necessário atuar com medidas que estimulem a mudança em relação à informação, prevenção e profilaxia dessas parasitoses, buscando adquirir e repassar conhecimentos. Nesse contexto, a educação ambiental no controle das enteroparasitoses tem se mostrado uma estratégia eficaz, com baixo custo e capaz de atingir resultados significativos e duradouros (ASOLU e OFOEZIE, 2003) e a escola como um centro de estudos e educação, deve observar os aspectos epidemiológicos das comunidades ao redor, e mapear os fatores de risco (SENNANUNES et al., 2001).

Bizerra e col. (1981) consideram que dados obtidos em trabalhos como este são fundamentais para o planejamento racional de eventuais programas de intervenção. Considera-se então que o processo educativo deve ser prioritário e contínuo nas ações de prevenção e qualidade de vida humana.

\section{CONCLUSÕES}

Este trabalho revelou um elevado índice de poliparasitismo, principalmente por protozoários. Inferese que a razão deste diagnostico esteja relacionado às condições sociais, econômicas e ambientais em que essas crianças são expostas, bem como, as características indivíduais. É importante ressaltar que a crescente eficácia da utilização de atividades didáticas/educativas envolvendo a ludicidade como ferramenta no processo educativo/profilaxio, é de grande valia, principalmente nas comunidades mais carentes, onde a educação ainda necessita de mais apoio governamental. É fato que em se tratando de crianças, a participação e a motivação por um determinado conhecimento, devem ser estimuladas através das brincadeiras, encenações, atividades dinâmicas, músicas, desenhos, arte e cultura em geral, podendo aprender e atuar como multiplicadores de informações dentro da escola e na sua casa com a sua família.

\section{REFERÊNCIAS}

ASOLU, S.O. \& OFOEZIE, I. E. The role of health education and sanitation in the control of helminthes infections. Acta Tropica, v. 86, n. 2, p. 283-94, 2003.

BARÇANTE, A.T.; CAVALCANTI, D.V.C; SILVA, G.A.V.; LOPES, P.B.; BARROS, R.F.; RIBEIRO,G. P.; NEUBERT,L.F.; BARÇANTE, J.M.P. Enteroparasitoses em crianças matriculadas em creches públicas do município de Vespasiano, Minas Gerais. Revista de Patologia Tropical, v. 37, p.33-42, 2008. 
BIZERRA, J. F.; GAZZANA, M. R.; COSTA, C. H.; MELLO, D. A.; MARSDEN, P. D. A survey of what people know about Chagas disease. World Health Forum, v.2, p.394-7, 1981.

BOTELHO, A.J. Relação entre infecções helmínticas, estado nutricional e desempenho cognitivo de escolares residentes em área endêmica. 106 f. Dissertação, Programa de Pós- Graduação em Enfermagem Escola de Enfermagem da Universidade Federal de Minas Gerais, 2008.

CASTRO, A. Z.; VIANA, J.D.C., PENEDO, A.A.; DONATELE, D.M. Levantamento das Parasitoses Intestinais em Escolares da Rede Pública na Cidade de Cachoeiro de Itapemirim - ES. NewsLab - edição 63, p. 102-5, 2004.

COELHO, J. C. V. Incidência de enteroparasitas em alunos do grupo escolar "Dr. Oswaldo Cruz", Curitiba, Paraná. Acta Biológica Paranaense, v. 4, n. 1/2, p. 3-12, 1975.

COURA, J.R.; WILlCOS, H.P.F.; TAVARES, A.M.; CASTRO, J.A.F.; FREITAS, A.D.; PÉREZ, E.P.; BORGES, L.L. Aspectos epidemiológicos, sociais e sanitários em áreas do Médio Solimões. II- Estudo de dois bairros periféricos da cidade de Coari e quatro localidades no Lago do Mamiá, Estado do Amazonas. Anais da Academia Nacional de Medicina, v.153, n.4, p.183-186, 1993.

CORREIA, A; BRANDÃO, D.S.; RIBEIRO, L.B. Estudo das parasitoses intestinais em alunos da $5^{\text {a }}$ Série do Colégio da Polícia Militar (CPM) de Feira de Santana-Bahia. Diálogos \& Ciência - Revista Eletrônica da Faculdade de Tecnologia e Ciências de Feira de Santana, v.3, n.6, 2005.

DE CARLI, G.; CANDIA, E.; NUNES, E.; SILVA, A.; MARTIN, R.; ANTUNES, I.; FARIAS, G.; LEIRIA, B.; CANDELA, M. H. Estudo de enteroparasitoses e das condições sócio econômicas e sanitárias das vilas periféricas de Porto Alegre, RS, Brasil, durante o período de 1965 a 1981. Caderno de Farmacologia, v. 5, n. 112, p. 73-92, 1989.

EVANGELISTA; KOMMA, M.D.; SANTOS, M.A. Prevalência de parasitos intestinais em Goiânia. Revista de Patologia Tropical, v. 1, n.1, p. 51-61,1972.

EZEAMAMA, A. E.; FRIEDMAN, J. F.; ACOSTA, L.P.; BELLINGER, D.C.; LANGDON, G.C.; MANALO, D.L.; OLVEDA, R.M.; KURTIS, J.D.; MCGARVEY, S.T. Helminth infection and cognitive impairment among Filipino children. American Journal of Tropical Medicine and Hygiene, v.72, n.5, p.540$548,2005$.

FORTES, B.P.M.D.; VALENCIA, L.I.O.; RIBEIRO, S.V.; MEDRONHO, R.A. Modelagem Geoestatística da infecção por Ascaris lumbricoides. Caderno de Saúde Pública, v.20, n.3, p.727 - 734, 2004.

GOMES DOS SANTOS, M.; MOREIRA, M.; MALAQUIAS, M.L.; SCHALL, V.T. Educação em saúde em escolas públicas de $1^{\circ}$ grau da periferia de Belo Horizonte, MG., Brasil. II. Conhecimentos, opiniões e prevalência de helmintíases entre alunos e professores. Revista do Instituto de Medicina. Tropical de São Paulo, v.35, n.6, p.573 - 9, 1993.

HADIDJAJA, P.; BONANG, E.; SUYARDI, M.A.; ABIDIN, S.A.; ISMID, I.S.; MARGONO, S.S. The effect of intervention met hods on nutritional status and cognitive function of primary school children infec ted with Ascaris lumbricoides. American Journal of Tropical Medicine and Hygiene, v.59, n.5, p.791-795, 1998.

HUGGINS, D.W.; MEDEIROS, L.B.; OLIVEIRA, E.R. Himenolepíase. Atualização e prevalência no hospital das Clinicas da UFPE. Revista de Patologia Tropical, v.22, n.1, p. 57-70, 1993.

MAIA, R.G. \& SCHIMIN, E.S. [s.d.] [Online] Ilustrações: recurso didático facilitador no ensino de Biologia. Homepage: http://www.diaadiaeducacao.pr.gov.br/portals/pde/arquivos/10824.pdf? PHPSESSID =200905061533 2531. Acesso em 03 de jan. 2013 . 
MELLO, D.A.; PRIPAS, S.; FUCCI, M.; SANTORO, M.C.; PEDRAZZANI, E.S. Helmintos intestinais: conhecimentos, atitudes e percepção da população. Revista de Saúde Pública, v.22, n.2, p.140-9, 1988.

MOURA, R.A.; WADA, C.S.; PURCHIO, A.; ALMEIDA, T.V. Técnicas de laboratório. $3^{\text {a }}$. ed. São Paulo: Ed. Atheneu, 2006.

NEVES, D.P. Parasitologia Humana. São Paulo: Atheneu, 2005.

NOKES, C.; GRANTHAM-MCGREGOR, S.M.; SAWYER, A.W.; COOPER, E.S.; BUNDY, D.A. Parasitic helminth infection and cognitive function in school children. Proceedings Biological Sciences, v.22, p.77$81,1992$.

PITTNER, E.; MORAES, I.F.; SANCHES, H.F.; TRINCAUS, M.R.; RAIMONDO, M.L.; MONTEIRO, M.C.M. Enteroparasitoses em Crianças de uma Comunidade Escolar na Cidade de Guarapuava, PR. Revista Salus-Guarapuava, v.1, n.1,p. 97-100, 2007.

PUPULIN, A.R.T.; GUILHERME, A.L.F.; ARAÚJO, S.M. Envolvimento de acadêmicos em programa integrado visando a melhoria das condições de vida de comunidades. Acta Scentiarium, v. 23, n. 3, p. 725729. 2000.

REY, L. Parasitologia. Rio de Janeiro: Guanabara Koogan, 2001. 731 p.

SAKTI, H.; NOKES, C.; HERTANTO, W.S.; HENDRATNO, S.; HALL, A.; BUNDY, D.A.; SATOTO. Evidence for an association between hookworm infection and cognitive function in Indonesian school children. Tropical Medicine and International Health, v.4, n.5, p.322-334, 1999.

SEIXAS, M.T.L.; SOUZA, J.N.; SOUZA, R.P.S.; TEIXEIRA, M.C.A.; SOARES, N. M. Avaliação da frequência de parasitos intestinais e do estado nutricional em escolares de uma área periurbana de Salvador, Bahia, Brasil. REVISTA DE PATOLOGIA TROPICAL, v. 40, n.4, p. 304-314, 2011.

SENNA -NUNES, M. S.; MELlO - SILVA, C. C.; BARBOSA G. L. S.; DIAS, B.; LORCA, F. O.; SIMÕES, L. G. Ações educativas para a prevenção de parasitoses aplicadas em escolas no município de Nova Iguaçu, RJ.,Brasil. XV Congresso Latino-americano de Parasitologia, São Paulo, out., 2001.

SILVA, E.F.; SILVA, E.B.; ALMEIDA,K.S.; SOUSA, J.J.N.; FREITAS,F.L.C. Enteroparasitoses em crianças de áreas rurais do munícipio de Coari, Amazonas, Brasil. Revista de Patologia Tropical, v. 38, n.1, p.35-43, 2009.

SIQUEIRA, R. V. \& FIORINI, J.E. Conhecimentos e procedimentos de crianças em idade escolar frente a parasitoses intestinais. Revista da Universidade de Alfenas, v.5, p.215-220, 1999.

UEMURA, A.N. \& CARVALHO, L. M. Os alunos de $6^{\mathrm{a}}$ a $8^{\mathrm{a}}$ séries do $1^{\mathrm{o}}$ grau frente aos conceitos básicos sobre parasitoses e suas implicações para saúde. Ciência e Cultura, v.41, n.7, p.702 - 8, 1989.

ULUKANLIGIL, M.; SEYREK, A.; ASLAN, G.; OZBILGE, H.; SULEYMAN, A. Environmental pollution with soil-transmitted helminthes in Sanliurfa, Turkey. Memórias do Instituto Oswaldo Cruz, v.96, n.7, p.903-909, 2001.

VINHA, C. Fundamentos e importância das campanhas contra os geo-helmintos no Brasil. Revista Brasileira de Malariología e Doenças Tropicais, v. 17, n.4, p.379-406, 1965. 